EPJ Web of Conferences 64, 02006 (2014)

DOI: $10.1051 /$ epjconf/ 20146402006

(C) Owned by the authors, published by EDP Sciences, 2014

\title{
Accretion in supergiant High Mass X-ray Binaries
}

\author{
Antonios Manousakis ${ }^{1,2, a}$, Roland Walter ${ }^{2}$, and John Blondin ${ }^{3}$ \\ ${ }^{1}$ Centrum Astronomiczne im. M. Kopernika, Bartycka 18, PL-00716 Warszawa, Poland \\ ${ }^{2}$ ISDC \& Observatoire de Genève, Université de Genève, ch. d'Ecogia 16, CH-1290 Versoix, Switzerland \\ ${ }^{3}$ Department of Physics, North Carolina State University, Raleigh, NC 27695-8202, USA
}

\begin{abstract}
Supergiant High Mass X-ray Binary systems (sgHMXBs) consist of a massive, late type, star and a neutron star. The massive stars exhibits strong, radiatively driven, stellar winds. Wind accretion onto compact object triggers X-ray emission, which alters the stellar wind significantly. Hydrodynamic simulation has been used to study the neutron star - stellar wind interaction it two sgHMXBs: i) A heavily obscured sgHMXB (IGR $J 17252$ - 3616) discovered by INTEGRAL. To account for observable quantities (i.e., absorbing column density) we have to assume a very slow wind terminal velocity of about $500 \mathrm{~km} / \mathrm{s}$ and a rather massive neutron star. If confirmed in other obscured systems, this could provide a completely new stellar wind diagnostics. ii) A classical sgHMXB (Vela $\mathrm{X}-1$ ) has been studied in depth to understand the origin of the off-states observed in this system. Among many models used to account for this observed behavior (clumpy wind, gating mechanism) we propose that self-organized criticality of the accretion stream is the likely reason for the observed behavior. In conclusion, the neutron star, in these two examples, acts very efficiently as a probe to study stellar winds.
\end{abstract}

\section{Introduction}

In supergiant High Mass X-ray Binaries (sgHMXBs) neutron stars are orbiting at a close distance of $\alpha \sim 1.5-2 \mathrm{R}_{*}$ from their companion stars. The donors, in these systems, are OB supergiants with mass loss rates of the order of $\sim 10^{-6} M_{\odot} \mathrm{yr}^{-1}$ and wind terminal velocities of $\sim 1500 \mathrm{~km} \mathrm{~s}^{-1}$. The neutron star accretes gas from the stellar wind and a fraction of the gravitational potential energy is converted into X-rays, ionizing and heating the surroundings. The X-ray emission can be used to investigate the structure of the stellar wind in situ [1].

The interactions between the neutron star and the stellar wind in Vela X-1 revealed that the wind of the massive star is heavily disrupted by the gravity and photoionization of the neutron star [2,3]. The heavily obscured sgHMXBs share some of the characteristics of the classical sgHMXBs. The main difference between classical and obscured sgHMXBs is that the latter ones are much more absorbed in the X-rays $\left(N_{H}>10^{23} \mathrm{~cm}^{-2}\right)$ on average, 10 times larger than in classical systems and well above the galactic absorption.

\footnotetext{
ae-mail: antonism@camk.edu.pl
} 


\section{Simulations \& Results}

\subsection{The simulations}

The VH $1{ }^{1}$ hydrodynamical code [2, 3] has been employed in order to study the interplay between the stellar wind and the compact object and compare it with the observational features of two supergiant sgHMXB systems $[4,5]$. Namely, to explain the origin of the obscuration of the heavily obscured eclipsing sgHMXB IGR $J$ 17252-3616 and to understand the origin of the X-ray off-states in the classical sgHMXB Vela X-1. In our simulations of sgHMXBs we account for: i) the gravity of the primary and of the neutron star, ii) the radiative acceleration of the stellar wind of the primary star, and iii) the suppression of the stellar wind acceleration in the Strömgren sphere of the neutron star. The simulations take place in the orbital plane, reducing the problem to two dimensions. In both cases we have assumed circular orbits $(e=0)$.

The code produces density and ionization $\left(\xi=L_{X} / n r_{n s}^{2}\right.$, where $L_{X}$ is the average X-ray luminosity, $n$ is the number density at the distance $r_{n s}$ from the neutron star [6]) maps that are stored. These allow to determine the simulated column density. As short time-scale variations occurs, we have calculated the time-averaged orbital phase resolved column density. The 3-D instantaneous mass accretion rate $\left(\dot{M}_{a c c}\right)$ onto the neutron star is also recorded. From the mass accretion rate, we can infer the instantaneous X-ray luminosity of the neutron star. The relaxation time is of the order of 0.5 orbits. The first couple of days of the simulations are therefore excluded from our analyses. In both simulations, the grid is centered on the center of mass. The boundary conditions are set in a way that matter is removed when it reached the cell including the neutron star. The boundary conditions at the radial outermost part of the mesh are characterized as outflow.

The winds of massive supergiant stars are radiatively driven by absorbing photons from the underlying photosphere, as described in CAK model [7]. However, regions in the stellar wind can differ from the predictions of the CAK/Sobolev approximation when instabilities are taken into account [8]. The velocity is described by the $\beta$-velocity law, $v=v_{\infty}\left(1-R_{*} / r\right)^{\beta}$ where $v_{\infty}$ is the terminal velocity and $\beta$ is the gradient of the velocity field. For supergiant stars, values for wind terminal velocities and mass-loss rates are in the range $v_{\infty} \sim 1500-3000 \mathrm{~km} \mathrm{~s}^{-1}$ and $\dot{M}_{\mathrm{w}} \sim 10^{-(6-7)} \mathrm{M}_{\odot} \mathrm{yr}^{-1}$, respectively [9].

A critical ionization parameter, above which the radiative force is negligible is defined. For $\xi>$ $10^{2.5} \mathrm{erg} \mathrm{cm} \mathrm{sec}^{-1}$, most of the elements responsible for the wind acceleration are fully ionized and hence the radiative acceleration force vanishes. The main effects of the ionization is the reduction of the wind velocity in the vicinity of the neutron star and the enhancement of the mass accretion rate onto the compact object.

\subsection{Obscured sgHMXB: IGR J17252-3616}

The obscured sgHMXB IGR $J 17252-3616$ is an eclipsing binary hosting a pulsar with $\mathrm{P}_{s} \sim 414 \mathrm{sec}$, an orbital period of $\mathrm{P}_{o} \sim 9.74$ days, and an orbital radius of $\alpha \approx 1.75 R_{*}$. Ground-based observations [10] showed that the donor star is likely a B0-5I or B0-1 Ia. Optical and IR observations confirmed the supergiant nature and showed prominent P-Cygni profile [11]. To explain the XMM-Newton and INT EGRAL observations, we [12, MW11] suggested that the wind terminal velocity of the system is relatively, low, of the order of $v_{\infty} \sim 500 \mathrm{~km} \mathrm{~s}^{-1}$.

In order to study this system, a computational mesh of 600 radial by 247 angular zones, extending from 1 to $\sim 15 \mathrm{R}_{*}$ and in angle from $-\pi$ to $+\pi$, has been employed. The grid structure is non-uniform, with cells size decreasing towards the neutron star. The maximal resolution, reached close to the neutron star, is $\delta R \sim 10^{10} \mathrm{~cm} \sim r_{a c c} / 3$

\footnotetext{
${ }^{1}$ http://wonka.physics.ncsu.edu/pub/VH-1/
} 

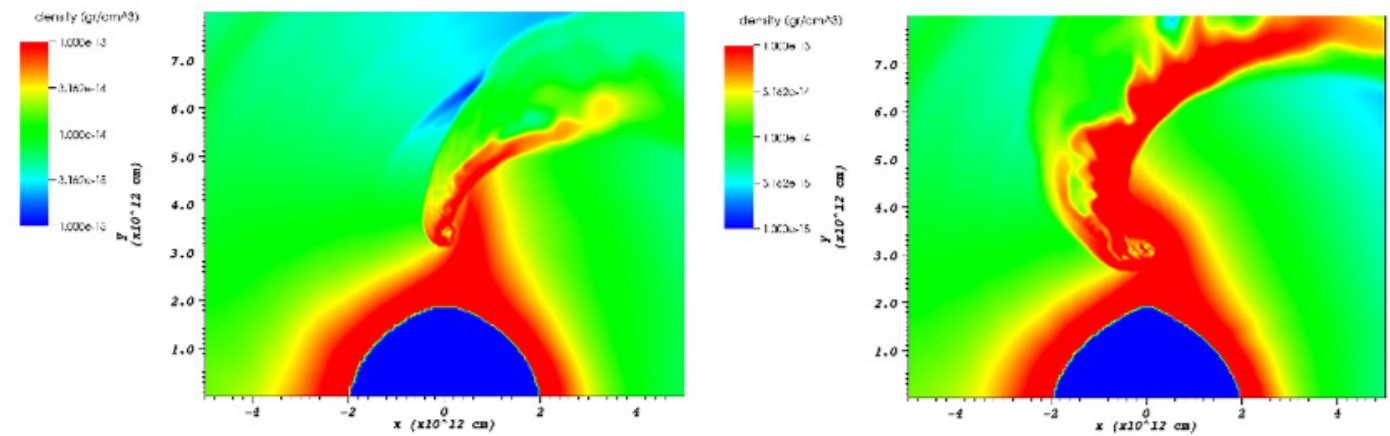

Figure 1. Density distribution (in gr $\mathrm{cm}^{-3}$; color bar) on the orbital plane after $\sim 3$ orbits. The wind terminal velocity is $v_{\infty} \approx 500 \mathrm{~km} \mathrm{~s}^{-1}$ and the mass-loss rate is $\dot{M}_{w} \approx 10^{-6} M_{\odot} \mathrm{y} \mathrm{r}^{-1}$. The mass of the neutron star scales from 1.5 (left) to $2.0 M_{\odot}$ (right). The color version of this figure is available on-line.

The appropriate modeling of stellar wind resulted in a smooth stellar wind with a terminal velocity of $v_{\infty} \approx 500 \mathrm{~km} \mathrm{~s}^{-1}$ and a mass loss rate of $\dot{M}_{w} \approx 10^{-6} M_{\odot} \mathrm{y} \mathrm{r}^{-1}$. This wind terminal velocity is about 2-3 times less than expected for an $\mathrm{OB}$ supergiant of the same spectral type and effective temperature. The effect of the gravity of the neutron star deflecting the accretion flow as can be seen in figure 1 between the left (for a neutron star mass of $1.5 \mathrm{M}_{\odot}$ ) and the right (for a neutron star mass of $2.0 \mathrm{M}_{\odot}$ ) panels. For a heavier neutron star, more gas will accumulate and the absorption will get stronger.

The heavier the neutron star, the more bended the accretion wake. This effect is revealed in the orbital phase dependency of the absorbing column density (see fig. 2; left panel), moving the position of the minimum to earlier phases. The histogram of the source observed light-curve (black) as well as the background (green), obtained with INTEGRAL through HEAVENS ${ }^{2}$ interface are shown in figure 2 (right panel). The inset panel shows the histogram derived from the simulated light-curve, and is well fit by a log-normal distribution. The convolution of the simulated data and the background is then compared with the source (blue curve). We can therefore estimate, in the frame of our model, that the mass of the neutron star is in the range $M_{N S}=1.75-2.15$.

The outcome of this work is described in Manousakis, Walter $\mathcal{E}$ Blondin [4]

\subsection{The classical sgHMXB Vela X-1}

Vela X-1 (=4U 0900-40) is a classical eclipsing super-giant High Mass X-ray Binary (sgHMXB). The system consists of an evolved B $0.5 \mathrm{Ib}$ supergiant (HD77581) and of a massive neutron star of $\mathrm{M}_{N S}=1.86 \mathrm{M}_{\odot}$ [13]. The neutron star orbits its massive companion with a period of about 8.9 days, in a circular orbit with a radius of $\alpha=1.76 R_{*}$ [14]. The stellar wind is characterized by a mass-loss rate of $\sim 4 \times 10^{-6} \mathrm{M}_{\odot} \mathrm{yr}^{-1}$ [15] and a wind terminal velocity of $v_{\infty} \approx 1700 \mathrm{~km} \mathrm{~s}^{-1}$ [16]. The X-ray luminosity is typically $\sim 4 \times 10^{36} \mathrm{erg} \mathrm{s}^{-1}$, although high variability can be observed. Recent studies on the hard X-ray variability of Vela X-1 have revealed a rich phenomenology. Flaring activity and short off-states have been observed [17]. Both flaring activity and off-states were interpreted as the effect of a strongly structured wind, characterizing the X-ray variability of Vela X-1 with a log-normal distribution, interpreted in the context of a clumpy stellar wind [18]. The quasi-spherical subsonic

\footnotetext{
${ }^{2}$ http://www.isdc.unige.ch/heavens/
} 

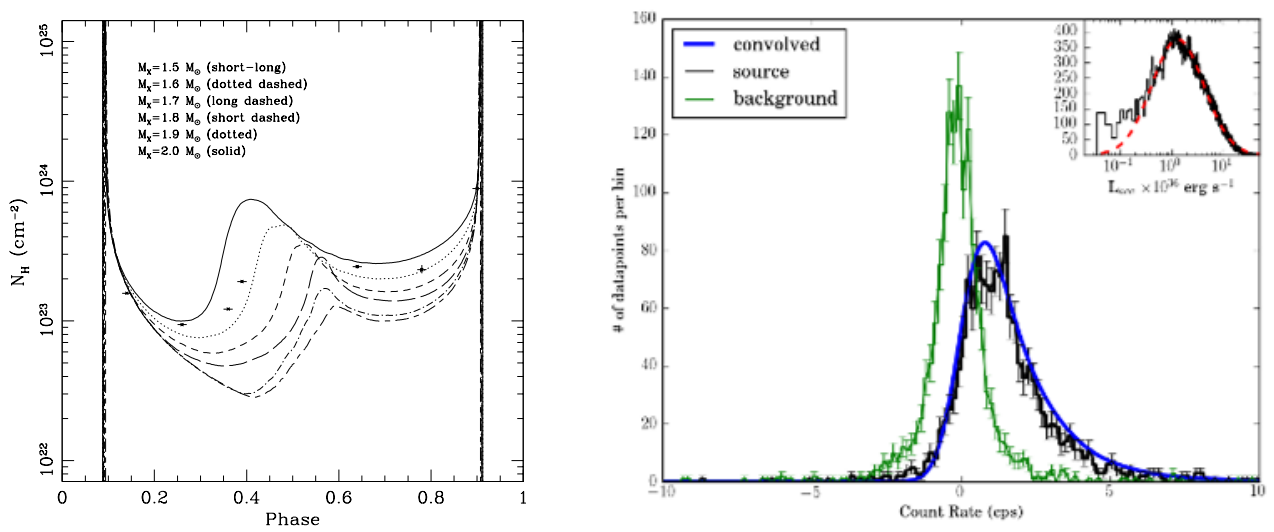

Figure 2. Left: Time-averaged simulated absorbing column density $\left(N_{H}\right)$ for various neutron star masses. The two limiting cases (short-long dashed and solid) are the results from figure 1. The data points are from MW11. Right: The histogram of observed light-curve (black), the background fluctuation (green), and the convolution of the simulated data with the latter (blue). The inset shows the corresponding distribution of the simulated. light-curve (black) with a log-normal fit (red dashed curve) The color version of this figure is available on-line.

accretion model $[19,20]$ is an alternative, predicting that the repeatedly observed off-states in Vela $\mathrm{X}-1$ are the result of a transition from the Compton cooling (higher luminosity) to radiative cooling (lower luminosity)

A computational mesh of 900 radial by 347 angular zones, extending from 1 to $\sim 25 \mathbf{R}_{*}$ and in angle from $-\pi$ to $+\pi$, has been employed. The grid is built in a non-uniform way in order to allow for higher resolution of $\sim 10^{-9} \mathrm{~cm}$ at the neutron star.

Our simulations predicts the formation of low density bubbles behind the bow shock, around the neutron star, resulting in the X-ray off-states. These bubbles are $\sim 10$ times larger than the accretion radius. When a bow shock appears, it moves away from the neutron star up to a distance of $\sim 10^{11}$ $\mathrm{cm}$ (see fig. 3). A low density bubble forms behind and starts expanding. It then gradually fall back and a stream of gas eventually reaches the neutron star and produces a new rise of the X-ray flux. The accretion stream can either move left-handed or right-handed. This 'breathing' behavior is quasi-periodic. This behavior is illustrated in figure 4 (left panel) with the simulated X-ray lightcurve over-plotted with a sinusoidal curve with a period of $6820 \mathrm{sec}$. The observed light-curves also show a quasi-periodic signal at $\sim 6800 \mathrm{sec}$ [17]. This modulation is related to the characteristic free-fall time of the low density bubble (radius of $\sim 10^{11} \mathrm{~cm}$ ) much longer than the accretion or magnetospheric radii.

We have constructed histograms of the observed and simulated light-curves (see fig. 4; right panel). The histograms are normalized to an integral equals to unity. All three distribution can be fit with the log-normal distribution characterized by a standard deviation $(\sigma)$ of 0.23 for INTEGRAL (blue) and 0.30 for RXTE (red), while the distribution is narrower for simulated lightcurve (black) having a standard deviations of 0.2. A minor excess can be seen at the lower end of the distribution at about $L_{X} \sim 10^{35} \mathrm{erg} \mathrm{s}^{-1}$. 


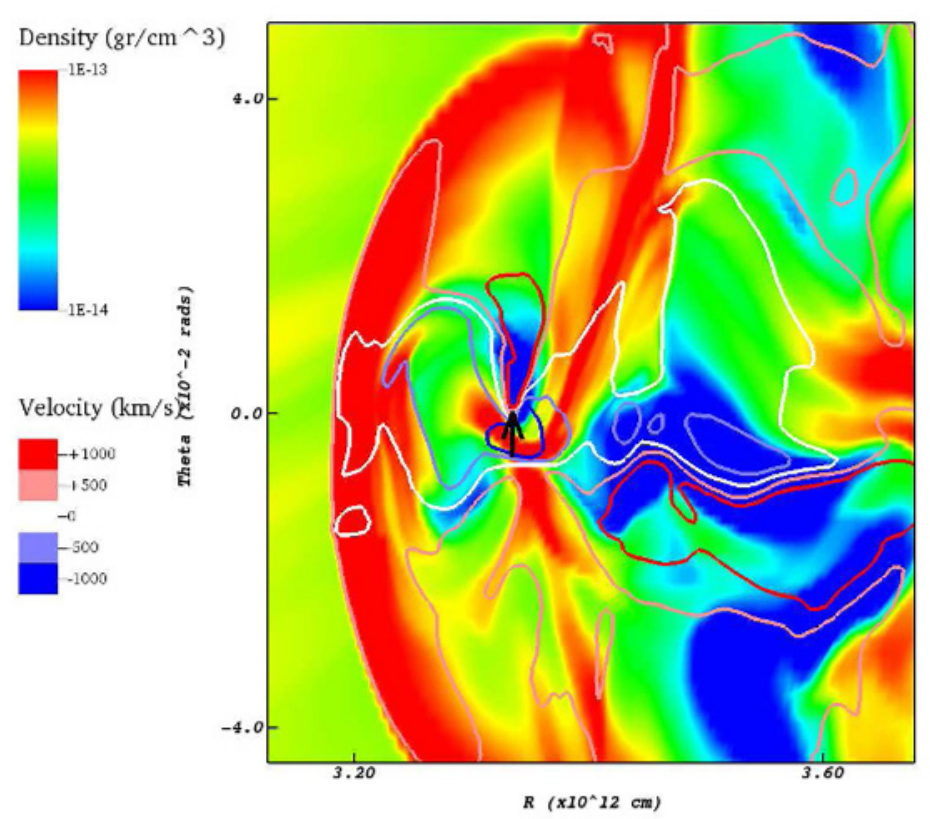

Figure 3. Density distribution (in $\mathrm{gr}^{-3}$ ) during the off-state. The over-plotted contours shows the radial velocities. The position of the neutron star is indicated by the black arrow. The color version of this figure is available on-line.
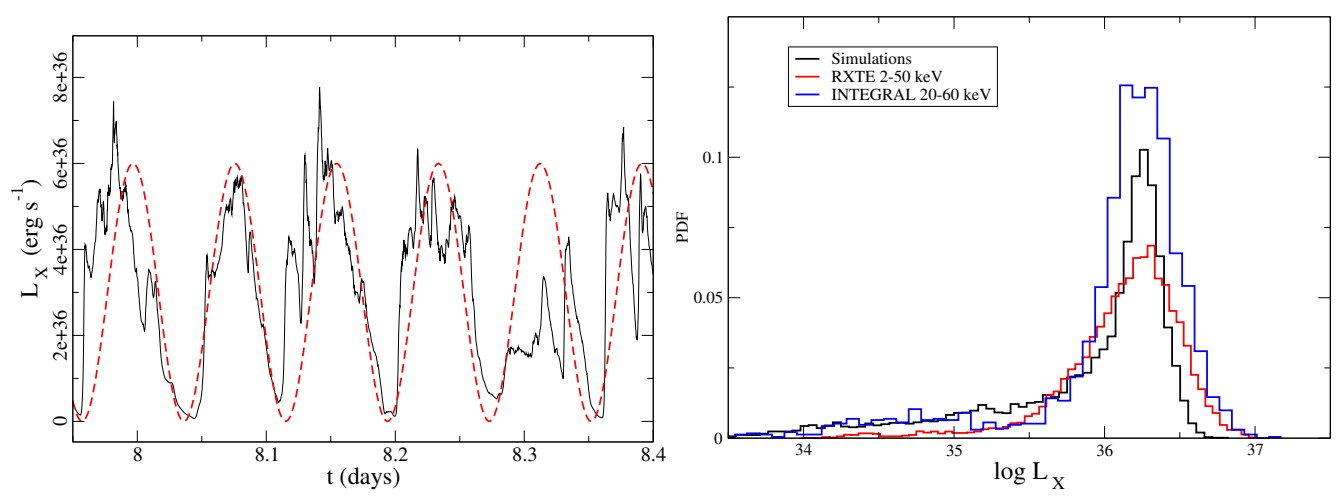

Figure 4. Left: Simulated light-curve of Vela X-1 together with a sine wave of $6820 \mathrm{sec}$ period (red dashed line). This quasi-periodic modulations was observed in Vela X-1 by [17]. The color version of this figure is available on-line. Right: The X-ray luminosity distribution of Vela X-1 from RXTE (2-50 keV; red) and INTEGRAL (20-60 keV; blue) observations together with that derived from the simulations (black). All the distributions are normalized to unity. 
The hydrodynamic simulations of Vela X-1 are sufficient to explain the observed behavior without the need for clumpy stellar wind or high magnetic fields and gating mechanisms. Self-organized criticality [21] of the accretion stream is enough to describe the observed variability.

The outcome of this work is described in Manousakis $\mathcal{F}$ Walter [5]

\section{Conclusion}

We have compared observed properties with the results of hydrodynamical simulations in two sgHMXB systems. Our main conclusions are as follows,

- The obscured sgHMXBs can bee understood in terms of low wind terminal velocities.

- The comparison between the hydrodynamical simulations with observations, in obscured sgHMXB, allow to constrain neutron star mass (and orbital radius), independently of dynamical estimates.

- In both simulations, we are able to produce log-normal distributions of the accretion rates. In addition, for Vela X-1 we are able to produce the X-ray off-states.

- Self-organized criticality of the accretion stream can explain the observed variability.

\section{References}

[1] R. Walter, J. Zurita Heras, A\&A 476, 335 (2007)

[2] J.M. Blondin, T.R. Kallman, B.A. Fryxell, R.E. Taam, ApJ 356, 591 (1990)

[3] J.M. Blondin, I.R. Stevens, T.R. Kallman, ApJ 371, 684 (1991)

[4] A. Manousakis, R. Walter, J.M. Blondin, A\&A 547, A20 (2012)

[5] Manousakis A. \& Walter, R., submitted to A\&A (2013)

[6] C.B. Tarter, W.H. Tucker, E.E. Salpeter, ApJ 156, 943 (1969)

[7] J.I. Castor, D.C. Abbott, R.I. Klein, ApJ 195, 157 (1975)

[8] S.P. Owocki, J.I. Castor, G.B. Rybicki, ApJ 335, 914 (1988)

[9] R. Kudritzki, J. Puls, ARA\&A 38, 613 (2000)

[10] A.B. Mason, J.S. Clark, A.J. Norton, I. Negueruela, P. Roche, A\&A 505, 281 (2009)

[11] S. Chaty, F. Rahoui, C. Foellmi, J.A. Tomsick, J. Rodriguez, R. Walter, A\&A 484, 783 (2008)

[12] A. Manousakis, R. Walter, A\&A 526, A62+, (MW11) (2011)

[13] H. Quaintrell, A.J. Norton, T.D.C. Ash, et al., A\&A 401, 313 (2003)

[14] L. Bildsten, D. Chakrabarty, J. Chiu, et al., ApJs 113, 367 (1997)

[15] F. Nagase, S. Hayakawa, N. Sato, K. Masai, H. Inoue, PASJ 38, 547 (1986)

[16] A.K. Dupree, H. Gursky, J.H. Black, et al., ApJ 238, 969 (1980)

[17] I. Kreykenbohm, J. Wilms, P. Kretschmar, et al., A\&A 492, 511 (2008)

[18] F. Fürst, I. Kreykenbohm, K. Pottschmidt, J. Wilms, et al., A\&A 519, A37+ (2010)

[19] N. Shakura, K. Postnov, A. Kochetkova, L. Hjalmarsdotter, MNRAS 420, 216 (2012)

[20] N. Shakura, K. Postnov, L. Hjalmarsdotter, MNRAS 428, 670 (2013)

[21] P. Bak, C. Tang, K. Wiesenfeld, Phys. Rev. A 38, 364 (1988) 